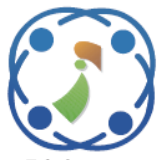

\title{
Brain Tumor Classification for MR Images Using Hybrid GLCM-LDTP-Le-Net Feature extraction and Bi-LSTM model
}

\author{
Madha Bramarambika ${ }^{1 *}$ \\ ${ }^{I}$ Telangana University, Dichpally, Nizamabad, India \\ * Corresponding author’s Email: mbambika@gmail.com
}

\begin{abstract}
The MRI is assisted by doctors to evaluate the tumors that help them for planning the required treatment. The physicians are dependent on the medical MRI diagnosis but that required lots of effort in finding out the tumor regions. The existing models speed up the scanning process using the MRI technique that reduced the burdens from radiologists but showed abnormality due to the presence of outliers when extracting hyperparameters. The proposed Hybrid GLCM-LDTP-Le-Net Feature extraction technique is developed by combining the Grey level covariance Matrix (GLCM), Local Direction Ternary Pattern (LDTP) and Le-Net features. The feature extraction technique results with hybrid parameters, which are applied to the Bidirectional Long Short Term Memory (Bi-LSTM) model to classify the brain MRI as a Malignant or Benign. The proposed hybrid feature extraction increases the accuracy of learned models by extracting the combined features from the input data. The proposed hybrid feature extraction obtained an accuracy of $97.88 \%$, better when compared with the existing CNN-based models' methods that obtained accuracy of $91 \%$.
\end{abstract}

Keywords: Benign, Brain tumor, Hybrid GLCM-LDTP-Le-Net feature extraction, Malignant, MRI images.

\section{Introduction}

Every sixth death in the world is due to cancer and the brain tumor stands in second place after cardiovascular diseases. Brain tumors will be the deadliest disease when compared among the distinct types of cancer because of their heterogeneous characters [1]. The tumor identification is important at an early step that classifies the brain tumor with a specific grade which shows improvement in the treatment. There are distinct kinds of brain tumors that are part of the deadliest forms which will be detected based on the features such as location, texture and shape of the tumour [2]. According to the world health organization (WHO), the malignancy grade in central nervous system, brain tumors are classified into different grades such as benign or Malignant). The brain tumor is effectively recognized by Magnetic Resonance Imaging (MRI) without any surgery in the brain [3, 4]. MRI imagining is a painfree and non-invasive medical imaging process used for executing excellent pictures of tumors [5]. MRI is widely considered to be the most useful technique for the detection of tumors because of its high-resolution property and classification on the brain image is effective. However, misdiagnosing the type of brain tumor leads to a significant problem that reduces the medical involvement to improve the survival chances among the patients [6]

Many attempts have been taken over the past years for diagnosing MR brain images for performing automated classification of the tumour. The existing methods performed classification based on the machine learning approaches that included supervised and unsupervised learning approaches [7]. The learning approaches occur through sequential stages initializing with pre-processing, feature extraction, and classification process that obtains final output [8]. Similarly, the segmentation process uses clustering approaches which was a challenging task [9]. Many studies have been examined in the machine learning techniques for brain tumour classification are discussed. With lots of effort, many types of research based on brain tumors were examined using smaller datasets. [10]. The existing 
models' utilized feature extraction techniques, where more irrelevant features were extracted that resulted in overfitting and complexity problems. The MR images are undergone for data augmentation and normalization process which leads to an extension of geometrical features. This phase of the general framework reduces the dimensionality of data by removing the redundant data. Thus, in the present research, a hybrid GLCM-LDTP-Le-Net Feature extraction is proposed where the GLCM functions characterizes texture features of an image that calculates the pair of pixels with similar values which specifies the spatial relationship among the image creates the GLCM. The LDTP model then extracts the statistical measures from the matrix. The coarse grid for stable codes generates higher level of features. Using these features, the Le-Net convolution extract spatial features will be straightforward learning the basics of CNNs runs on the GPU for the extraction of features. The proposed hybrid GLCM-LDTP-Le-Net feature extraction obtained accuracy of $98.88 \%$, sensitivity of $96.42 \%$, and specificity of $96.44 \%$ better when compared with the generative adversarial network that obtained accuracy of $88.82 \%$, sensitivity of $92.17 \%$, and specificity of $81.81 \%$.

The structure of the paper is as given follows: Section 2 describes the literature survey; Section 3 describes the proposed method. Section 4 present the results and discussion. The conclusion and future work of this research paper is given in Section 5.

\section{Literature review}

The existing researches involved in brain tumor classification is as follows:

Chenjie [11] developed a brain tumor classification model using pairwise generative adversarial networks (GANs) for molecular-based enlarged training dataset. The developed GAN-based pairwise model performed 2 staged course training strategies for learning the features of glioma using GAN. The results showed that the developed model classified the molecular subtypes based on the isocitrate dehydroge component into Benign or malignant. However, the developed model failed when multiple datasets applied for data augmentation process to classify the important subtypes as that required information of age, survival years etc., of the patients.

Alhassan and zainon [12] performed brain tumor classification using MRI images based on hard Swish RELU activation function with CNN model. The developed model effectively utilized a histogram descriptor for the detection of contour and edge features to obtain better classification results. The extracted features were fed as input for $\mathrm{CNN}$ which were classified as glioma, meningioma, and pituitary tumors based on the hard swish activation function, which improved the learning speed of the model. The time period required for the model training was varied from one system to another required improvement in accuracy.

Kumar [13] performed a multi-class brain tumor classification using residual network (ResNet) and global average pooling (GAP). The developed model overcame the problem of overfitting and vanishing gradient which were the demerits of the deep networks. The developed model used ResNet-50GAP that evaluated the efficiency of the model using a 3 tumor brain MRI 3064 dataset images. However, the model was computationally expensive and further required reduction in the computational time.

Narmatha [14] developed a hybrid fuzzy brain-storm optimization algorithm for performing the classification of brain MRI images. The developed model utilized brain tumor segmentation showed complexity to perform. The developed fuzzy brain storm optimization approach performed automated segmentation and classification that concentrated mainly on the centers of clusters which gave them a high priority for the classification. However, the developed brain storm optimization approach was utilized only for segmenting and classifying the images but failed to operate for different processes.

Goryawala [15] developed a T1-weighted and T2-weighted subtraction MRI for glioma visualization and grading maps. These visualizations and grading maps were generated by $\mathrm{T} 1$ and $\mathrm{T} 2$ weighted images subtraction process. The developed model generated the normal generation of white matter, enhancement of tumor, and necrotic region that provided complete representation of tumor regions and these were used for tissue region separately. However, they lacked in 3D ROI that introduced confusion during the selection of features lead to lower performances.

A. Aldhahab [16] developed a stacked sparse auto encoder and soft-max classifier for classifying the brain MRI tumour images. The neural network consists of two types of neural network such as stacked sparse auto encoder (SSA) and soft-max classifier (SMC). Thus, the two regularization function are utilized for SA training, L2 weight regularization and sparsity regularization that controlled the hidden layer neurons for firing out. However, the L2-weight regularization failed to reduce the overfitting effects that can improves the SA performances further. 


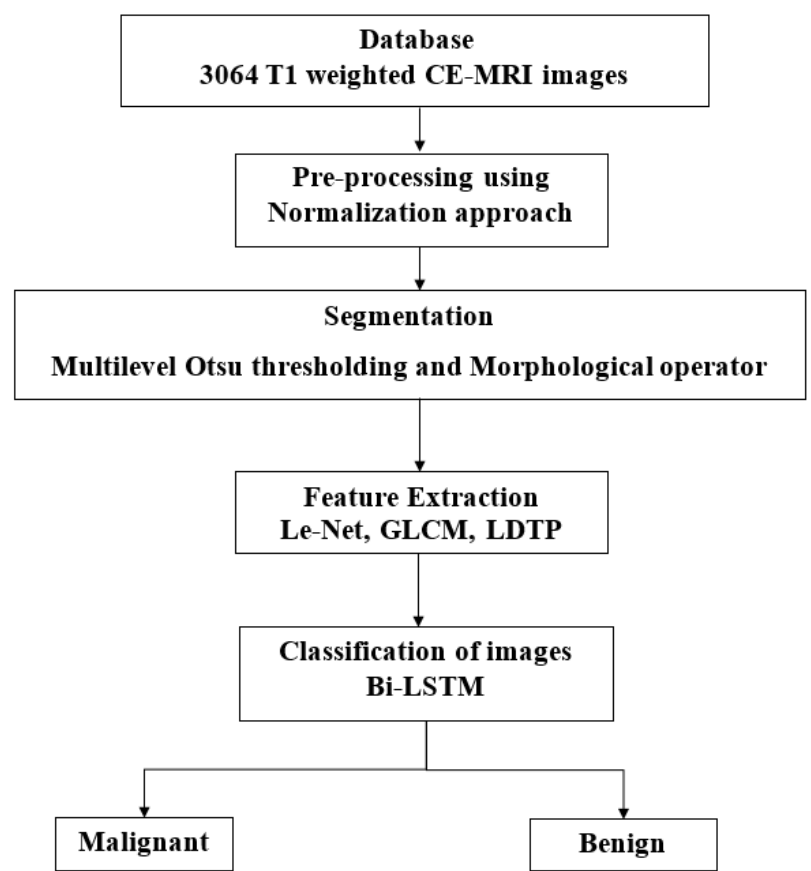

Figure. 1 Block diagram of the proposed GLCM-LDTPLe-Net Feature extraction method

Tjahyaningtijas [17] developed the En-CNN model for brain tumour classification based on the MRI images. The developed model used the brain tumour binary classification that characterized the MR multi-sequence images that are obtained based on the deep learning approach. The model was dependent on the En-CNN architecture that showed complexity problem. Thus, the developed model consisted of VGG-16 with seven CNN that considered max-pooling was facilities with the hyper parameter tuning.

Fouad [18] developed brain tumour using whale optimization algorithm (WOA) with ensemble learning classifier. The developed approach utilized haar discrete wavelet transforms with histogram of oriented gradients (HDWT-HOG) features formed a hybrid model. The feature descriptors based on the local gradients on the MR image based on the shape information. The WOA played an important role for the reduction of the numbers of number of features from HDWT-HOG reached up to 120 features. However, the developed model was required to be extended for combined computer vision and deep learning for achieving more obtaining reliable results.

Rao and Lingappa [19] performed MRI based brain tumour detection using hybrid segmentation and deep learning classification. The developed model used hybrid kernel based fuzzy C-means clustering - convolutional neural network (Hybrid KFCM-CNN) method for classification. The Hybrid KFCM- CNN method used T1 -W CEMRI database for validation of results. However, an effective bio inspired approach was required further for performance improvement analysed the proposed method performance.

Kumar [20] developed genetic algorithm for performing brain tumour segmentation and classification. The developed model utilized adaptively regularized kernel-based fuzzy c-means (ARKFCM) algorithm resized $256 \times 256$ in the pre processing stage. The developed ARKFCM was highly flexible for machine learning technique located the object as a complex template. However, the hybrid feature extraction required to be performed on the image which was segmented improved the feature subsets.

\section{Proposed method}

The block diagram of the proposed hybrid GLCM-LDTP-Le-Net feature extraction method is shown in Fig. 1.

The presented block diagram consists of dataset block, pre-processing, segmentation, feature extraction, and classification.

\subsection{Brain tumour MRI database}

The brain tumor dataset consists of $3064 \mathrm{~T} 1$ weighted contrast-enhanced images of 233 patients who suffer from any of the three stages such as 1 st stage, 2nd stage, and 3rd stage of the brain tumor. The dataset images are taken from the hospitals of China like Nanfang hospital and general hospital has the record which starts from 2005. The dataset images were applied for the normalization process and thus helped the model for performing the best. Brain MRI source of image dataset is used to perform the segmentation of MRI. The test images are acquired with TR which utilizes 5850 and Echo time as 130. The FLAIR weighted MRI was acquired to the spectra MR machine with 135 images in total with 9 slices of image per patient and each of the images having FOV of $200 \mathrm{~m}$. The real dataset including the brain MRI has a $512 \times 512$ pixel size which is converted to grayscale before processing. In the proposed method, the large dataset is used to overcome the problem by using a small dataset for misclassification in existing CNN methods. Fig. 2 shows the sample MRI brain tumor images present in the database [16].

\subsection{Pre-processing using normalization process}

The MRI images usually consist of a dark large area which is not a brain image and it requires an accelerating process to remove those unwanted 


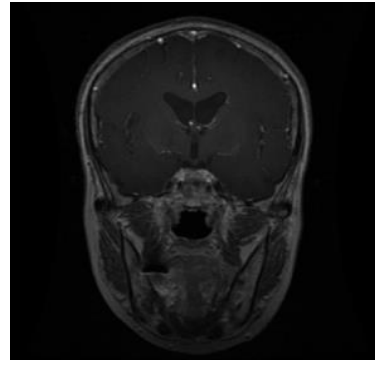

(a)

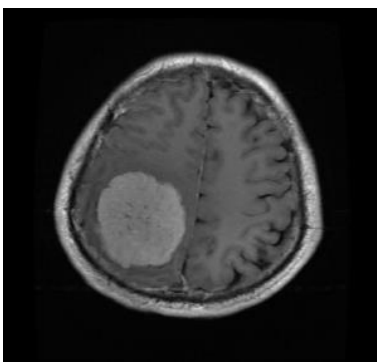

(b)

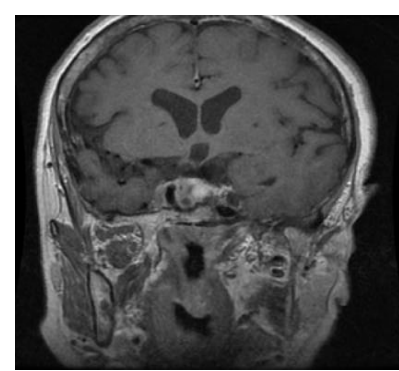

(c)

Figure. 2 Input images: (a) Glioma, (b) Meningioma, and (c) Pituitary

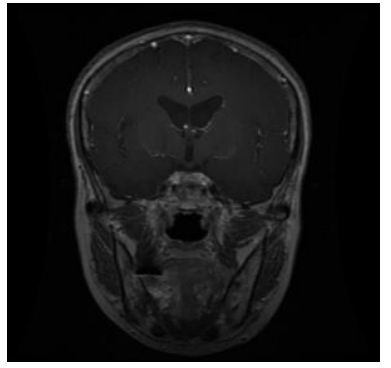

(a)

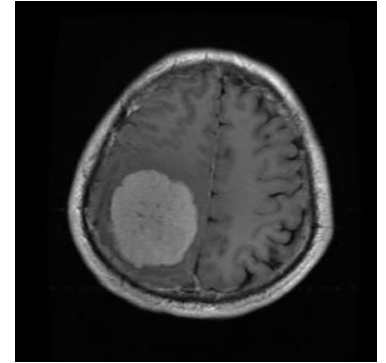

(b)

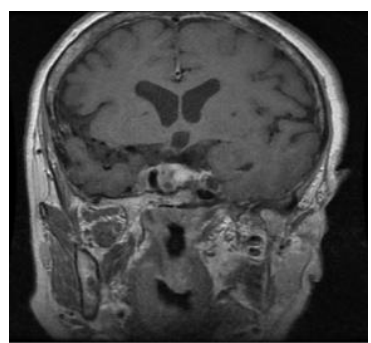

(c)

Figure. 3 Pre-processed image: (a) Glioma, (b) Meningioma, and (c) Pituitary

lesions from MRI images. The speckle noise is created when magnetic fields are cycled ON and OFF during MRI scanning and these unwanted noises are removed using normalization which is shown in Fig. 3.

\subsubsection{Normalization:}

The MRI image generated by MRI scanners includes noise and non-uniform intensity distribution, so the image normalization technique is used to solve such problem during MRI normalization. The image normalization process is used to change the normal intensity values of pixels to more familiar intensity values of pixels. General formula of color normalization technique is defined in Eq. (1).

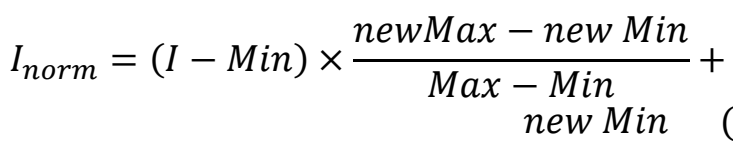

\subsection{Segmentation using morphology operation and multilevel thresholding no masking}

The segmentation of the masking region is done by using a region growing technique, but still, the unwanted regions from the segmented brain MRIs are needs to be removed. The morphology operation is used in this research to remove unwanted regions based on the shapes and sizes of an input image. The segmented region with structural element is given as input image to morphological operations and it produces the same size output image without losing any important qualities. To perform such a function, Multilevel-otsu threshold algorithm performs pixel separation of an input image into different classes that separate gray levels based on the intensity values. Several thresholds are calculated using multi-otsu that determines classes of the desired number [17]. The main reason for using multilevel otsu thresholding and morphological operator techniques is to eliminate unwanted regions even after masking using morphology operation. Morphological operations are performed for processing functions based on shapes and sizes.

The weighted parameter values within the class probabilities are calculated using the Eq. (2).

$$
\begin{aligned}
& q_{1}(t)=\sum_{i=1}^{t} P(i), \\
& q_{2}(t)=\sum_{i=t+1}^{I} P(i), \\
& q_{n}(t)=\sum_{i=I+t+1}^{n} P(i)
\end{aligned}
$$

The threshold value ranges from 1 to $t$

$q_{1 ., n}$ is weighted class within pixel probabilities $P$ of the foreground and background.

The class means are given in Eq. (3).

$$
\begin{aligned}
& \mu_{1}(t)=\sum_{i=1}^{t} \frac{i P(i)}{q_{1}(t)}, \mu_{2}(t) \\
& =\sum_{i=t=1}^{I} \frac{i P(i)}{q_{2}(t)}, \ldots ., \mu_{n}(t) \\
& =\sum_{i=n}^{t} \frac{i P(i)}{q_{n}(t)}
\end{aligned}
$$

where, $\mu_{1}$ and $\mu_{2}$ are the average gray level values

An input image that is having a structuring element is used for performing morphological operations and obtains an output image without 


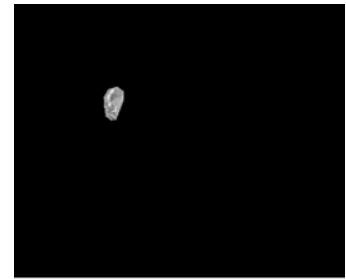

(a)

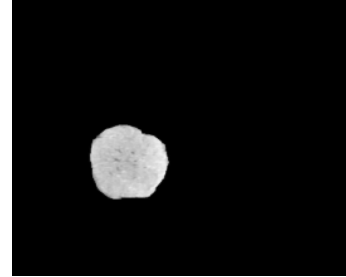

(b)

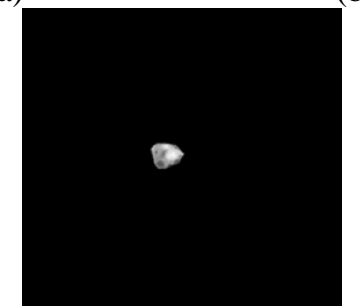

(c)

Figure. 4 Segmented image: (a) Glioma, (b) Meningioma, (c) Pituitary

losing its properties. The morphological operation is performed for each pixel of an input image that corresponds to the neighborhood pixels. The shape and size of an image is chosen based on the neighborhood pixels and morphological operation is performed for constructing specific shapes for an input image. The image obtained after segmentation is presented in Fig. 4.

\subsection{Feature extraction}

An efficient hybrid features extraction method combines the features and the features obtained are fed as an input for the Bi-LSTM classifier for classifying the MRI brain tumor images.

\subsubsection{GLCM}

Among various texture-based feature extraction techniques, the researchers considered GLCM technique as it can operate based on second-order statistics for identifying the textural relationship between pixels. To operate, GLCM used only pixels and identifies the frequency of combined pixel brightness values. A matrix with a same number of columns and rows is described as gray values in MR images by expressing the properties of GLCM. According to the two specified pixels frequency, the matrix elements will be constructed, where the pairs of both pixels are changing that depends on their neighborhood. The two features of GLCM considered are Correlation and Homogeneity.

\subsubsection{Homogeneity}

The closeness of the elements in gray level matrix distribution is used to find the homogeneity features. It considers the homogeneous texture regions classify quantitatively and the texture features are calculated using Gabor filtering scale. The image of glaucoma is divided into homogeneous texture regions and features related to texture local spatial statistics are calculated using Gabor filtering. The GLCM calculates for four directions $\left(0^{0}, 450,900\right.$ or 1350$)$ with a feature vector size of four. The general formula to calculate the homogeneity of MR image is represented in Eq. (4).

$$
\text { Homogeneity }=\sum_{i=0}^{n-1} \sum_{j=0}^{n-1} \frac{P_{i j}}{1+(i-j)^{2}}
$$

\subsubsection{Correlation}

The correlation technique calculates the dependency of grey level of neighboring pixels linearly in an image. The accurate measurement of three and two dimension measurements changes in the images of brain. The Eq. (5) represent correlation for tumor image.

$$
\text { Correlation }=\sum_{i=0}^{n-1} \sum_{j=0}^{n-1} P_{i j} \frac{(i-\mu)(j-\mu)}{\sigma^{2}}
$$

Where, mean is represented as ' $\mu$ ', the standard deviation is ' $\sigma$ ', the gray levels of brain tumor image is represented as ' $n$ ', $(i, j)$ is the position of the pixel $P(i j), P_{i j}$ is the co-occurrence matrix.

\subsubsection{Local direction ternary pattern}

A vast amount of information for texture image is lost due to binary coding in the existing patterns. The traditional techniques namely local ternary pattern (LTP), local binary pattern (LBP), and other LBPlike texture descriptors used the coding strategy of bit strings that leads to ignoring the most useful information of the neighborhood. In an image, the micro-level information of spots, edges and other local features are encoded by these LBP-like methods using intensity information around every pixel. Moreover, all directions of the images are treated equally using the LDP operator and directional information will sign of response by LDP technique. A new coding scheme is designed to avoid this drawback by integrating both the concepts of LDP and LTP. According to the contrast information for encoding and local derivative variations, the pertinent properties are inherited from both compact encoding of directional pattern features in the proposed local texture modeling. Only one type of compass masks is used in both LDN and LDN operators, where two various compass masks namely 2nd derivative Gaussian mask and frei-chen masks are incorporated by the LDTP technique. Therefore, the edge responses are computed by encoding the image 
texture using eight directions based on two compass masks in the LDTP operators.

\subsubsection{LeNet}

It is the representative of an early convolution neural network $(\mathrm{CNN})$ model that possesses the units of $\mathrm{CNN}$ including convolutional layer, pooling layer and fully connection layer that lays on the basement for further development in CNN. The LeNet-5 has seven layers including the input and other layers which are used for parameter training. The Le-Net models that are pre-trained on images are good at detecting high-level features like edges, patterns, etc.

\subsection{Classification using Bi-LSTM}

The output values obtained from the GLCM, LDTP, and LeNet are used and in the next step classification of images for the detection and identification of benign and malignant is performed. Each of the classifiers has two significant modes one is training and other is testing modes. The Bi-LSTMs are the extension of LSTM that improves the model performances based on the classification problems that occurred in the sequence. All the generated time steps of the input sequences are available and $\mathrm{Bi}$ LSTM trains two model instead of only one LSTM on the input sequence. The Bi-LSTM will calculate the sequence from the opposite direction up to the forward hidden sequence and then again the backward hidden sequence. The encoded vector forms the concatenation up to the final forward and backward outputs. It calculates the input sequences $x=\left(x_{1}, x_{2}, \ldots, x_{n}\right)$ from an opposite direction towards the forward hidden sequence $\vec{h}_{t}=$ $\left(\vec{h}_{1}, \vec{h}_{2}, \ldots, \vec{h}_{n}\right)$ and the backward sequence $\overleftarrow{h}_{t=}\left(\overleftarrow{h}_{1}, \overleftarrow{h}_{2}, \ldots, \overleftarrow{h}_{n}\right)$. An encoded vector is $y_{t}$ which is formed by the process of concatenation of the output obtained from the forward and the backward outputs $y_{t}=\left[\vec{h}_{t}, \overleftarrow{h_{t}}\right]$ which are calculated using Eq. (6 to 8 ).

$$
\begin{aligned}
& \vec{h}_{t}=\sigma\left(W_{\vec{h} x} x_{t}+W_{\vec{h} \vec{h}} \vec{h}_{t-1}\right) \\
& \overleftarrow{h}_{t}=\sigma\left(W_{\vec{h} x} x_{t}+W_{\vec{h} \vec{h}} \overleftarrow{h}_{t-1}\right) \\
& y_{t}=W_{y \vec{h}} \vec{h}_{t}+W_{y \vec{h}} \overleftarrow{h}_{t}
\end{aligned}
$$

Where $y_{t}$ represents the outputs obtained for $\left(y_{1}, \ldots, y_{n}, \ldots, y_{t}\right)$

$\sigma$ is the function of backward and forward processes.
The obtained sequence will be ranging between the 0 and 1 values and this sequence is considered as an input for the problem each number is provided to the time. A binary label ( 0 or 1$)$, for each input, will be associated. The output values are assigned as 0 and once the cumulative sum for all the input values are obtained in sequence, then a threshold is generated among the output values ranging from 0 to 1 . In this study, a deep learning classifier is undertaken for classifying the benign and malignant for glioma, pituitary, Meningioma's using a deep neural network.

\section{Results and discussion}

The proposed model evaluated the results of classification using accuracy, F1-score, recall, precision to indicate the clear generalization of the model that imbalanced the MRI images dataset. The simulations are conducted using an intel core i7 processor with $2 \mathrm{GHz} \mathrm{CPU}$ utilization time and 48 GB of RAM. The training data is fed to the classifier and evaluated with respect to the testing data. The achieved performances such as Accuracy, sensitivity, specificity, and F-measure are described as follows:

\subsection{Performance measures}

The proposed method performance is evaluated using the following parameters:

\section{- Accuracy:}

Accuracy is defined as the ratio of correctly predicted to the total number of observations. The accuracy is calculated by using Eq. (9).

$$
\operatorname{Accuracy}(\%)=\frac{(T P+T N)}{(T P+T N+F P+F N)} \times 100
$$

\section{- Sensitivity}

Sensitivity is the calculation that measures the ratio of correctly determined positives and it is defined in Eq. (10).

$$
\text { Sensitivity }(\%)=\frac{T P}{T P+F N} \times 100
$$

\section{- Specificity}

Specificity is the calculation that measures the ratio of correctly determined negatives and it is defined in Eq. (11).

$$
\text { Specificity }(\%)=\frac{T P}{T N+F P} \times 100
$$


Table 1. Results for the proposed hybrid feature extraction method

\begin{tabular}{|c|c|c|c|c|c|}
\hline Features & Classifier & Accuracy (\%) & Sensitivity (\%) & Specificity $(\%)$ & F-score $(\%)$ \\
\hline \multirow[b]{5}{*}{ LDTP } & ANN & 83.01 & 80.35 & 72.99 & 87.44 \\
\hline & RNN & 89.51 & 84.56 & 80.43 & 61.33 \\
\hline & $\mathrm{CNN}$ & 91.08 & 83.59 & 72.30 & 85.16 \\
\hline & LSTM & 80.59 & 84.98 & 76.60 & 89.04 \\
\hline & Bi LSTM & 91.98 & 87.10 & 85.55 & 91.88 \\
\hline \multirow[b]{5}{*}{ GLCM } & ANN & 75.43 & 82.26 & 66.73 & 88.69 \\
\hline & RNN & 91.07 & 89.91 & 87.35 & 63.35 \\
\hline & $\mathrm{CNN}$ & 84.34 & 89.52 & 80.85 & 84.48 \\
\hline & LSTM & 79.53 & 82.93 & 86.04 & 90.04 \\
\hline & Bi LSTM & 93.45 & 92.41 & 87.58 & 93.75 \\
\hline \multirow[b]{5}{*}{ Lenet } & ANN & 82.56 & 66.45 & 89.13 & 89.54 \\
\hline & RNN & 88.54 & 90.31 & 88.14 & 62.96 \\
\hline & $\mathrm{CNN}$ & 90.40 & 91.17 & 87.20 & 85.62 \\
\hline & LSTM & 74.39 & 78.94 & 71.75 & 88.82 \\
\hline & Bi LSTM & 93.65 & 94.24 & 94.01 & 92.65 \\
\hline \multirow[b]{5}{*}{ Hybrid } & ANN & 86.44 & 63.38 & 94.53 & 90.21 \\
\hline & RNN & 91.52 & 91.79 & 87.90 & 94.00 \\
\hline & $\mathrm{CNN}$ & 95.27 & 92.22 & 93.34 & 93.64 \\
\hline & LSTM & 95.05 & 95.22 & 95.62 & 88.60 \\
\hline & Bi LSTM & 98.88 & 96.44 & 96.42 & 96.05 \\
\hline
\end{tabular}

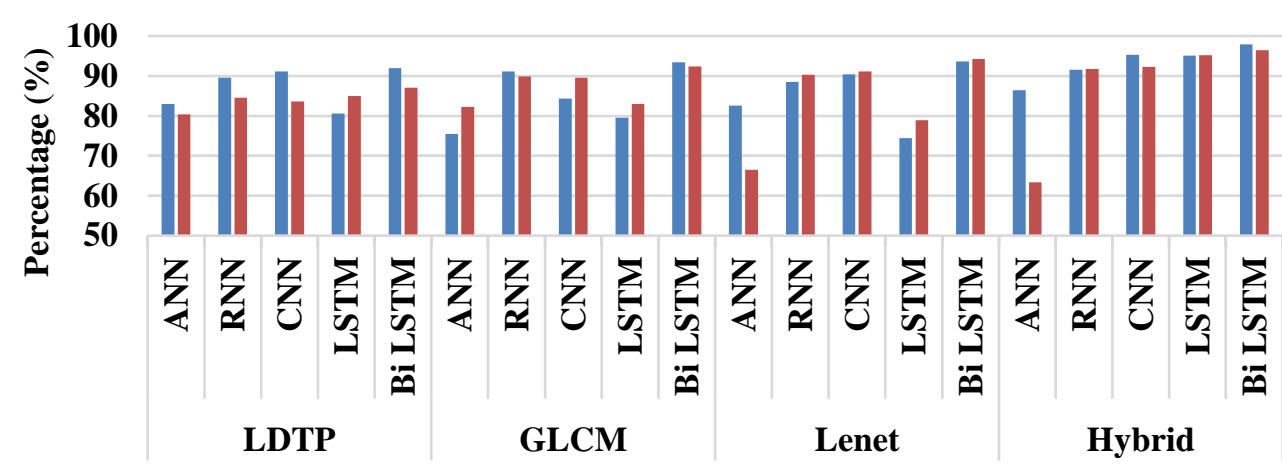

- Accuracy $\square$ Sensitivity

Figure. 5 The results obtained in terms of accuracy and sensitivity

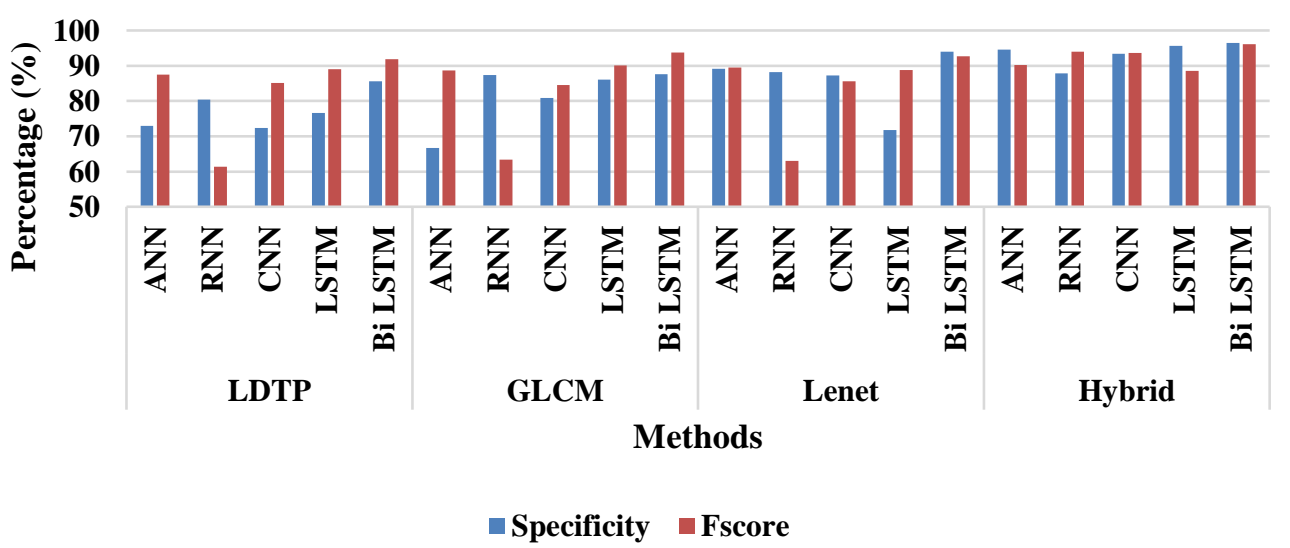

Figure. 6 The results obtained in terms of specificity and F-score 
Table 2. Comparative analysis

\begin{tabular}{|c|c|c|c|c|c|}
\hline Author & Dataset & Method & $\begin{array}{l}\text { Accuracy } \\
(\%)\end{array}$ & $\begin{array}{l}\text { Specificity } \\
(\%)\end{array}$ & $\begin{array}{l}\text { Sensitivity } \\
(\%)\end{array}$ \\
\hline Chenjie Ge[11] & \multirow{10}{*}{$\begin{array}{c}3064 \mathrm{~T} 1 \\
\text { weighted } \\
\text { contrast- } \\
\text { enhanced } \\
\text { images }\end{array}$} & $\begin{array}{c}\text { Generative Adversarial } \\
\text { Network }\end{array}$ & 88.82 & 92.17 & 81.81 \\
\hline Afnan M. Alhassan [12] & & $\begin{array}{c}\text { swish-based RELU- } \\
\text { CNN }\end{array}$ & 98.6 & - & - \\
\hline R Lokesh Kumar [13] & & $\begin{array}{l}\text { ResNet-50 and global } \\
\text { average pooling }\end{array}$ & 97.08 & - & - \\
\hline $\begin{array}{l}\text { C. Narmatha } \\
{[14]}\end{array}$ & & $\begin{array}{c}\text { Fuzzy brain-storm } \\
\text { optimization algorithm }\end{array}$ & 93.85 & - & 95.77 \\
\hline M. Goryawala [15] & & $\begin{array}{l}\text { T1-weighted and T2- } \\
\text { weighted Subtraction }\end{array}$ & 90.9 & & \\
\hline Ahmed Aldhahab et al [16] & & $\begin{array}{l}\text { Stacked Sparse Auto- } \\
\text { Encoder and Soft-max } \\
\text { Classifier }\end{array}$ & 94 & - & - \\
\hline $\begin{array}{c}\text { Hapsari Peni Agustin } \\
\text { Tjahyaningtijas et al [17] }\end{array}$ & & En-CNN & 97 & - & - \\
\hline Amal Fouad et al [18] & & $\begin{array}{l}\text { WOA based Ensemble } \\
\text { Learning Classifier }\end{array}$ & 96.4 & - & - \\
\hline $\begin{array}{c}\text { Sudheesh Kannur Vasudeva } \\
\text { Rao et al [19] }\end{array}$ & & Hybrid KFCM-CNN & 97.33 & - & - \\
\hline Proposed method & & $\begin{array}{c}\text { Hybrid GLCM-LDTP- } \\
\text { Le-Net feature } \\
\text { extraction }\end{array}$ & 98.88 & 96.42 & 96.44 \\
\hline
\end{tabular}

\section{- F1-measure}

The harmonic mean of recall and precision is defined as F1-measure, which is shown in Eq. (12).

$$
F 1-\text { measure }(\%)=\frac{2 \times \text { Precision } \times \text { Recall }}{\text { Precision }+ \text { Recall }} \times 100
$$

\subsection{Quantitative analysis}

Table 1 shows the results obtained for the proposed hybrid feature extraction method in terms of accuracy, sensitivity, specificity and F score. GLCM was utilized in the present research work obtained accuracy of $76.86 \%$, precision of $81.32 \%$, recall of $89.88 \%$, MCC of $87.12 \%$, and F-score of $78.98 \%$. However, with the large dimensionality data obtained from the GLCM's were sensitive towards the size and texture of the image samples.

Thus, the number of gray levels was reduced and the LDTP features utilized were sensitive for the image rotation. They produced long histograms that lead to a decrease in the distinctiveness required for large storage and textural information. Fig. 5 shows results obtained in terms of accuracy and sensitivity. Thus it extracted limited spatial support and was small enough to show highly sensitive to noise and blurring thus obtained accuracy, sensitivity, specificity, and F score of 55.81\%, 59.19\%, 72.48\%,
$57.69 \%, 67.18 \%$. Fig. 6 shows the results obtained in terms of specificity and F-score.

Similarly, the hybrid features that were fed for the Bi-LSTM classifier for the classification tends to give a much better classification accuracy of $93.65 \%$ because of used desirable features. From the results, it is clear that hybridizing these features will improve performances in terms of accuracy, sensitivity, specificity, and f-score.

The Bi-LSTM model classified accurately, the stages of cancer using glioma, pituitary, and menigiomas images and obtained accuracy of $98.88 \%$, Sensitivity of $96.44 \%$, specificity of $96.42 \%$, and F-score of $96.05 \%$ which was better when compared to the existing models.

\subsection{Comparative analysis}

Table 2 shows the comparative analysis for the existing and the proposed methods in terms of accuracy, sensitivity, and precision.

The existing model GAN [11] failed to obtain better results when applied for multiple datasets showed lower accuracy of $88.82 \%$. Similarly, swishbased RELU-CNN [12] was utilized for training varied from one system to another because of variations in the setup resulted in an accuracy of 98.6\%. Similarly, [13] utilized ResNet-50 and GAP obtained accuracy of 97.08 as it was computationally expensive and required a reduction in the 
computational time. Similarly, glioma visualization and grading maps [15] lead to lower performances accuracy of $90.9 \%$ because of selection of irrelevant features. The stacked sparse auto-encoder and softmax classifier [16] obtained accuracy of $94 \%$ as it failed to reduce the overfitting because of L2-weight regularization. The en-CNN [17] obtained accuracy of $97 \%$ that showed moderate accuracy values and WOA based ensemble learning classifier [18] obtained accuracy of $96.4 \%$ that still required improvement in the reliability of the model. The hybrid KFCM-CNN [19] obtained $97.33 \%$ showed complexity in the system. Whereas the proposed hybrid feature extraction model was designed that automatically segmented the tumour regions and from the segmented regions, the relevant features were extracted to overcome the problem of overfitting. The proposed hybrid feature extraction obtained an accuracy of $98.88 \%$, sensitivity of $96.344 \%$, and F-score of $96.42 \%$ and also it increases training strategies when compared with the existing CNN-based models methods.

\section{Conclusion}

Currently, the research work performs learning approaches through sequential stages by initializing with pre-processing, feature extraction process, and classification process that obtains final output. The existing models have examined various machinelearning techniques to classify brain tumours and they face challenges during the classification of a small dataset and training irrelevant features, resulted in misclassification. Therefore, the proposed model used a large dataset for the classification that was rarely available. The small datasets used for the proposed hybrid feature extraction research are automatically performed by segmentation where the relevant features were extracted to overcome the over-fitting problem. The proposed method shows a $2 \%$ improvement in accuracy when compared with the existing $\mathrm{CNN}$ technique. However, in the future, complexity is created in the $\mathrm{CNN}$ network due to more number of layer parameters that should be overcome by integrated models.

\section{Conflicts of interest}

The authors declare no conflict of interest.

\section{Author contributions}

The paper background work, conceptualization, methodology, Dataset collection, implementation, result analysis and comparison, preparing and editing draft, visualization, the supervision, review of work and project administration, have been done by first author.

\section{References}

[1] M. Sajjad, S. Khan, K. Muhammad, W. Wu, A. Ullah, and S. W. Baik, "Multi-grade brain tumor classification using deep CNN with extensive data augmentation", Journal of Computational Science, Vol. 30, pp. 174-182, 2019.

[2] Z. N. K. Swati, Q. Zhao, M. Kabir, F. Ali, Z. Ali, S. Ahmed, and J. Lu, "Brain tumor classification for MR images using transfer learning and finetuning", Computerized Medical Imaging and Graphics, Vol. 75, pp. 34-46, 2019.

[3] N. Ghassemi, A. Shoeibi, and M. Rouhani, "Deep neural network with generative adversarial networks pre-training for brain tumor classification based on MR images", Biomedical Signal Processing and Control, Vol. 57, pp. 101678, 2020.

[4] H. H. Sultan, N. M. Salem, and W. A. Atabany, "Multi-Classification of BrainTumor Images Using Deep Neural Network", IEEE Access, Vol. 7, pp. 69215-69225, 2019.

[5] K. Kaplan, Y. Kaya, M. Kuncan, and H. M. Ertunç, "Brain tumor classification using modified local binary patterns (LBP) feature extraction methods", Medical Hypotheses, p. 109696, 2020.

[6] I. E. Kaya, A. Ç. Pehlivanlı, E. G. Sekizkardeş, and T. Ibrikci, "PCA basedclustering for brain tumor segmentation of T1w MRI images", Computer Methods and Programs in Biomedicine, Vol. 140, pp. 19-28, 2017.

[7] S. A. A. Ismael, A. Mohammed, and H. Hefny, "An enhanced deep learningapproach for brain cancer MRI images classification using residual networks", Artificial Intelligence in Medicine, Vol. 102, p. 101779, 2020.

[8] G. Tamilmani and S. Sivakumari, "Early detection of brain cancer using association allotment hierarchical clustering", International Journal of Imaging Systems and Technology, Vol. 29, pp. 617-632, 2019.

[9] S. Maharjan, A. Alsadoon, P. W. C. Prasad, T. A. Dalain, and O. H. Alsadoon, "Anovel enhanced softmax loss function for brain tumour detection using deep learning", Journal of Neuroscience Methods, Vol. 330, p. 108520, 2020.

[10] S. Deepak and P. M. Ameer, "Brain tumor classification using deep $\mathrm{CNN}$ features via transfer learning", Computers in Biology and Medicine, Vol. 111, p. 103345, 2019. 
[11] C. Ge, I. Y. H. Gu, A. S. Jakola, and J. Yang, "Enlarged training dataset by pairwise gans for molecular-based brain tumor classification", IEEE Access, Vol. 8, pp. 22560-22570, 2020.

[12] A. M. Alhassan and W. M. N. W. Zainon, "Brain tumor classification in magnetic resonance image using hard swish-based RELU activation function-convolutional neural network", Neural Computing and Applications, pp.1-13, 2021.

[13] R. L. Kumar, J. Kakarla, B. V. Isunuri, and M. Singh, "Multi-class brain tumor classification using residual network and global average pooling", Multimedia Tools and Applications, Vol. 80, No. 9, pp. 13429-13438, 2021.

[14] C. Narmatha, S. M. Eljack, A. A. R. M. Tuka, S. Manimurugan, and M. Mustafa, "A hybrid fuzzy brain-storm optimization algorithm for the classification of brain tumor MRI images", Journal of Ambient Intelligence and Humanized Computing, pp. 1-9, 2020.

[15] M. Goryawala, B. Roy, R. K. Gupta, and A. A. Maudsley, "T1-weighted and T2-weighted Subtraction MR Images for Glioma Visualization and Grading", Journal of Neuroimaging, Vol. 31, No. 1, pp. 124-131, 2021.

[16] A. Aldhahab, S. Ibrahim, and W. B. Mikhael, "Stacked Sparse Autoencoder and Softmax Classifier Framework to Classify MRI of Brain Tumor Images", International Journal of Intelligent Engineering and Systems, Vol. 13, No. 3, pp. 268-279, 2020.

[17] H. P. Tjahyaningtijas, D. J. Rumala, C. V. Angkoso, N. Z. Fanani, J. Santoso, A. D. Sensusiati, P. M. V. Ooijen, I. K. Purnama, and M. H. Purnomo, "Brain Tumor Classification in MRI Images Using En-CNN", International Journal of Intelligent Engineering and Systems, Vol. 14, No. 4, pp. 437-451, 2021.

[18] A. Fouad, H. M. Moftah, and H. A. Hefny, "Brain Diagnoses Detection Using Whale Optimization Algorithm Based on Ensemble Learning Classifier", International Journal of Intelligent Engineering and Systems, Vol. 13, No. 2, pp. 40-51, 2020.

[19] S. K. Rao and B. Lingappa, "Image Analysis for MRI Based Brain Tumour Detection Using Hybrid Segmentation and Deep Learning Classification Technique", International Journal of Intelligent Engineering and Systems, Vol. 12, No. 5, pp. 53-62, 2019.

[20] V. V. Kumar, K. S. Krishna, and S. Kusumavathi, "Genetic Algorithm Based Feature Selection Brain Tumour Segmentation and Classification",
International Journal of Intelligent Engineering and Systems, Vol. 12, No. 5, pp. 214-223, 2019.

[21] S. Bhuvaji, A. Kadam, P. Bhumkar, S. Dedge, and S. Kanchan, Brain Tumor Classification (MRI) Dataset. Available online: https://www.kaggle.com/sartajbhuvaji/braintumor-classification-mri (accessed on 1 August 2020).

[22]S. Ravi and A. M. Khan, "Morphological operations for image processing: understanding and its applications", In: Proc. of 2nd National Conference on VLSI, Signal processing \& Communications NCVSComs, pp. 1-6, 2013. 